\title{
New era of regenerative medicine for cardiovascular diseases
}

\section{Hung-Fat Tse}

Cardiology Division, Department of Medicine, Queen Mary Hospital and Research Centre of Heart, Brain, Hormone and Healthy Ageing, Li Ka Shing Faculty of Medicine, The University of Hong Kong, Hong Kong, HKSAR, China

The increasing life expectancy in many parts of the world has led to an epidemiologic transition in the leading causes of death from infectious diseases and acute illness to chronic illness related to organ or tissue degeneration. For example, chronic non-communicable diseases, including cardiovascular diseases, chronic respiratory diseases and cancer now account for an estimated $80 \%$ of total mortality and $70 \%$ of disability-adjusted life-years lost in China (1). Despite the recent advances in medical and surgical therapies, a large number of patients with cardiovascular diseases remain severely symptomatic with poor clinical outcomes. Many currently untreatable cardiovascular disorders arise from disease process due to significantly loss of cardiomyocytes that do not otherwise regenerate. As a result, stem cell therapy has been explored as potential treatment to limit the progression of diseases or to regenerate damaged heart in patients with different cardiovascular diseases. Indeed, stem cell therapy was conceptualised more than a decade ago in the treatment of acute myocardial infarction (2). The articles in this Theme issue of Thrombosis and Haemostasis tackle several of the important topics in cardiovascular regeneration using stem cell therapy.

\section{Correspondence to:}

Hung-Fat Tse, MD, PhD

Cardiology Division, Department of Medicine

The University of Hong Kong, Queen Mary Hospital

Hong Kong, China

Tel.: +852 2855 3598, Fax: +852 28186304

E-mail: hftse@hkucc.hku.hk

\section{Financial support:}

This study was supported by Research Grants Council of Hong Kong, General Research Fund (No. HKU 7594/05M, HKU 7769/08M); Outstanding Researcher Award 2007-2008 (H.F.T); and Collaborative Research Fund of Hong Kong Research Grant Council (HKU 8/CRF/09).

Received: June 4, 2010

Accepted: June 4, 2010

Prepublished online: June 10, 2010

doi:10.1160/TH10-06-0347

Thromb Haemost 2010; 104: 4-5
Based on the initial encouraging results in the experimental studies, numerous clinical studies in recent years have been shown that cell-based therapy can improve symptoms and cardiac function, and limit infarct size and adverse ventricular remodelling in patients with acute myocardial infarction, chronic myocardial ischaemia and congestive heart failure. Siu et al. (3) present a critical overview on the current status, including those data from the randomised clinical trials on the use stem cell therapy for myocardial regeneration. Despite the promising initial clinical results, the beneficial effects are at best modest, and several major issues, such as the optimal timing, cell types and mode of delivery need to be addressed in the ongoing clinical trials. Furthermore, additional strategies to improve cell survival and engraftment should also be developed to overcome the potential hurdles related to cell-based therapy.

One of the major limitations of clinical success of stem cell therapy is the low cell retention and engraftment after transplantation. Accurate methods for cell tracking as well as quantification of cell survival are needed to determine the optimal method to enhance cell retention and engraftment. In addition to conventional laboratory techniques, novel in vivo imaging methods have been developed and can provide serial noninvasive assessment of cell fate. Ransohoff and $\mathrm{Wu}(4)$ describe the basic principles and techniques of radionuclide imaging, magnetic resonance imaging, bioluminescence imaging, and fluorescence imaging for assessing cell engraftment, discuss their strength and critically discuss their limitations. The development of these techniques to track cell fate and viability not only can provide important insight into the stem cell biology after transplantation, but also help to evaluate new techniques, such as genetic modification, bioengineering and cell preconditioning to optimise cell engraftment (3).

The other important obstacle for cardiac regeneration with stem cell therapy is the ability to replace any major loss of cardiomyocytes after the initial insult. For example, more that one billion of cardiomyocytes died after an acute myocardial infarction. Currently, adult stem cells, such as bone marrow, skeletal myoblast and mesenchymal stem cells are the most common cell source for cardiac regeneration in clinical trials as they have no risks of immune rejection with autologous transplantation, non-tumour forming, and have no ethical obstacle. However, they are restricted in their renewal and differentiation potential to become functional cardiomyocytes. In contrast, human pluripotent stem cells include embryonic stem cells (hESCs) and recently induced pluripotent stem (iPS) cells, via reprogramming of somatic cells can propagate indefinitely while maintaining their normal karyotypes and pluripotency to differentiate into all cell types. K. R. Boheler reviews (5) the stem cell biology of these human pluripotent cells and their potential therapeutic applications. Insights into the characteristics of these human pluripotent stem cells offers hope to improve efficacy for cardiac differentiation, and thus their therapeutic applications for cardiac regeneration. Nevertheless, the potential clinical applications of these pluripotent stem cells for cardiovascular therapies remain challenging. Kong et al. (6) discuss the functional and structural properties of cardiomyocytes derived from these human pluripotent stem cells, and highlighted the potential issues related to their immature phenotypes, such as calcium handling and electrophysiological properties. Therefore, new techniques to drive cardiac differentiation and maturation could ultimately enable the application of these human pluripotent stem cells to regenerate damaged myocardium.

Finally, the generation of human iPS cells has circumvented some of the potential technological, social and ethical issue related to hESC. Lian et al. (7) provide a perspective on the potential application of iPS cell platform for diagnosis, drug screening and cell-based therapy. Conceptually, 
iPS cell technology should provide an unlimited supply of even specified cells, not limited to cardiomyocyte, but also hepatocytes, beta-cells and neurons etc. for tailored made patients-specific cell-based therapy for treatment of various human diseases, and to develop human cell based platform for drugs screening. On the other hand, significant challenges, such as risk of viral vector and low efficacy during reprogramming as well as the potential for tumour formation remain to be overcome before the translation of iPS cell technology into clinical practice. In summary, it is an- ticipate that regenerative medicine will play an ever more prominent role in the new era of modern cardiovascular therapies; however, significant challenges must be overcome before that promise is realised.

\section{References}

1. Yang G, Kong L, Zhao W, et al. Emergence of chronic non-communicable diseases in China. Lancet 2008; 372: 1697-1705.

2. Orlic D, Kajstura J, Chimenti S, et al. Bone marrow cells regenerate infarcted myocardium. Nature 2001; 410: 701-705
3. Siu CW, Liao SY, Liu Y, et al. Stem cells for myocardial repair. Thromb Haemost 2010; 104: 6-12.

4. Ransohoff KJ, Wu JC. Advances in cardiovascular molecular imaging for tracking stem cell therapy. Thromb Haemost 2010; 104: 13-22.

5. Boheler KR. Pluripotency of human embryonic and induced pluripotent stem cells for cardiac and vascular regeneration. Thromb Haemost 2010; 104: 23-29.

6. Kong M, Fadi A, Li RA. Translational potential of human embryonic and induced pluipotent stem cells for myocardial repair: Insights from experimental models. Thromb Haemost 2010; 104:30-38.

7. Lian Q, Chow Y, Esteban MA, et al. Future perspective of induced pluripotent stem cell for diagnosis, drug screening and treatment of human diseases. Thromb Haemost 2010; 104: 39-44. 